\title{
Category scales of sweetness are consistent with sweetness-matching data
}

\author{
ROBERT L. McBRIDE \\ Commonwealth Scientific and Industrial Research Organization (CSIRO), Division of Food Research \\ Sydney, N.S.W., Australia
}

\begin{abstract}
Category scales were obtained for the sweetness of sucrose, fructose, and glucose, with care being taken to preclude methodological bias. These category scales were then used to predict the outcome of scale-free sweetness matching with the same three sweeteners. The matching concentrations predicted from the category scales were found to agree well with those actually obtained in several sweetness-matching studies.
\end{abstract}

Psychophysicists have yet to reach a consensus on the response measure to be used in direct scaling. The two most commonly used techniques, magnitude estimation and category rating, provide scales that are nonlinearly related. As Weiss (1981) notes, a literature survey reveals magnitude estimation to be the more popular technique; still, the debate continues, particularly in the chemical senses (Pangborn, 1981). Resolution of this issue requires a rigorous validity criterion against which various scaling methods can be checked.

The advent of functional measurement analysis (Anderson, 1970, 1972, 1981, chap. 5, 1982, chap. 1), with its reliance on factorial stimulus designs and algebraic integration models, appears to offer such a criterion. In functional measurement analysis, success of the algebraic integration model also validates the response scale. Anderson (e.g., 1972) has emphasized that functional measurement is essentially neutral in the debate between the response measures of magnitude estimation and category rating; but, although rating methods usually meet the requirements of a linear response scale, magnitude estimation usually fails.

In taste, a certain amount of psychophysical work has involved the use of a scale-free matching paradigm. This is especially true of sweetness measurement. A reference sweetener is selected, usually sucrose, and the subject is required to select that concentration of another sweetener which produces equivalent sweetness. By repeating this procedure at a number of reference concentrations, and with different matching stimuli, information on relative sweetness can easily be obtained (Lichtenstein, 1948; Pangborn, 1963; Yamaguchi, Yoshikawa, Ikeda, \& Ninomiya, 1970; Cameron, Note 1; Dahlberg \& Penczek, Note 2). Thus, the matching method sidesteps the scaling problem; however, it can, in fact, be used as a cross-check on the response scale.

Requests for reprints should be sent to R. L. McBride, CSIRO Division of Food Research, P. O. Box 52, North Ryde, N.S.W. 2113 , Australia.
If the sweetness-matching task is unbiased, and if category rating is a valid response technique (as the functional measurement approach suggests), then sweetness scales established by category rating should be consistent with the results of matching studies. The purpose of the present study is to check this prediction.

Category scaling is commonly regarded as being susceptible to several sources of methodological bias, the most problematical being the nonlinear stimulus spacing bias (Poulton, 1979; Stevens \& Galanter, 1957). But an iterative technique has been suggested as a means of removing this effect (Pollack, 1965; Stevens \& Galanter, 1957). In this procedure, subjects are first presented with a number of stimuli, arbitrarily spaced, and the psychophysical function generated therefrom is then used to determine the stimulus spacing for the next test. This procedure is repeated until the stimulus spacing suggested by the psychophysical function is the same as that used to obtain it (Pollack, 1965, gives details of the technique). With stimulus spacing bias eliminated, the investigator can lay claim to a "pure" category scale (Stevens \& Galanter, 1957). Frequency effects are of less concern, since they can be nullified by presenting all stimuli an equal number of times; and the linear range (centering) bias is unlikely to be a problem unless the psychophysical study is specifically concerned with the establishment of cutoff points (see McBride, 1982; Poulton, 1979).

The task in the present study was to derive pure category scales for the sugars sucrose, fructose, and glucose. These stimuli are natural sweeteners, free from sidetastes (Schiffman, Reilly, \& Clark, 1979); besides, they have been commonly used in matching studies, and consequently there is ample data available for the crosscheck on response-scale validity.

\section{METHOD}

\footnotetext{
Subjects

Subjects, who participated voluntarily, were male and female employees of the CSIRO Food Research Laboratory. All had
} 
had some previous experience in sensory testing. A panel of 24 was used for each experiment, but the panel composition altered slightly between experiments.

\section{Stimuli}

Stimuli, which consisted of reagent-grade compounds in distilled water, were always made up at least $24 \mathrm{~h}$ before testing to preclude the possibility of mutarotation effects (Cameron, Note 1). Stimulus temperature was approximately $20^{\circ} \mathrm{C}$, and stimulus volume was $30 \mathrm{ml}$.

For sucrose, there were three separate parts to the experiment. In Part 1, the stimulus concentrations were $.0625, .1250$, .2500 , and $.5000 \mathrm{M}$ (geometric/log spacing); in Part 2, they were $.0625, .0947, .1436, .2177, .3299$, and $.5000 \mathrm{M}$ (log spacing); and, in Part 3 , the initial concentrations were $.0625, .2083$, .3542 , and $.5000 \mathrm{M}$ (arithmetic spacing). The stimulus range was the same in each case.

For D-fructose, the initial concentrations were $.0972, .1944$, .3888 , and $.7776 \mathrm{M}$ (geometric spacing); for D-glucose, they were $.1500, .3000, .6000$, and $1.2000 \mathrm{M}$ (geometric spacing).

\section{Response Scale}

The response scale consisted of 13 categories, indicated by a column of 13 short lines, with five equidistant verbal descriptors-"Extremely sweet" labeling line 13; "Very sweet," 10 ; "Moderately sweet," 7; "Slightly sweet," 4; and "No sweetness at all," line 1. Stimulus end-anchors were not presented, nor were the categories numbered on the response sheet. The subject indicated a judgment by marking the appropriate line.

\section{Procedure}

Determination of the category scale for sucrose has been described previously (McBride, 1983). Parts 1 and 2 each consisted of two replicate sessions, the sessions within each part being held on consecutive days. Stimuli were presented simultaneously; subjects were required to taste and expectorate each in the order specified, and to rate each sweetness on the 13-point scale. Rinsing with distilled water (also at $20^{\circ} \mathrm{C}$ ) was mandatory between stimuli and the rinse volume was not restricted. In Part 1, all 24 possible permutations of presentation order were used, whereas in Part 2 the order was randomized. The procedure in Part 3 was similar to that in Part 1, except that the iterative procedure was employed: The psychophysical function obtained from the initial session suggested revised concentrations for the second session on the following day, and so on, until, at the fourth session, the suggested concentrations converged.

For fructose and glucose, this same iterative technique was employed. In both cases, the assessment consisted of three sessions, held on consecutive days.

\section{RESULTS AND DISCUSSION}

The mean category ratings ( $\pm 2 \mathrm{SE}$ ) for sucrose from Parts 1 and 2, and from the final session of the iterated Part 3, are given in Figure 1. (These data have been published in McBride, 1983, and are shown here again to allow easy comparison with the fructose and glucose data.) Over the concentration range used, there is close approximation to a straight line in a semilog plot (Fechner's law), and the ratings were fitted against log sucrose concentration by the method of least squares.

The first point of note is the good reproducibility of the category scaling. Mean ratings for the extreme stimuli, which were common to all three parts, barely changed between parts. The error bars show that response variability remained reasonably constant, regard-

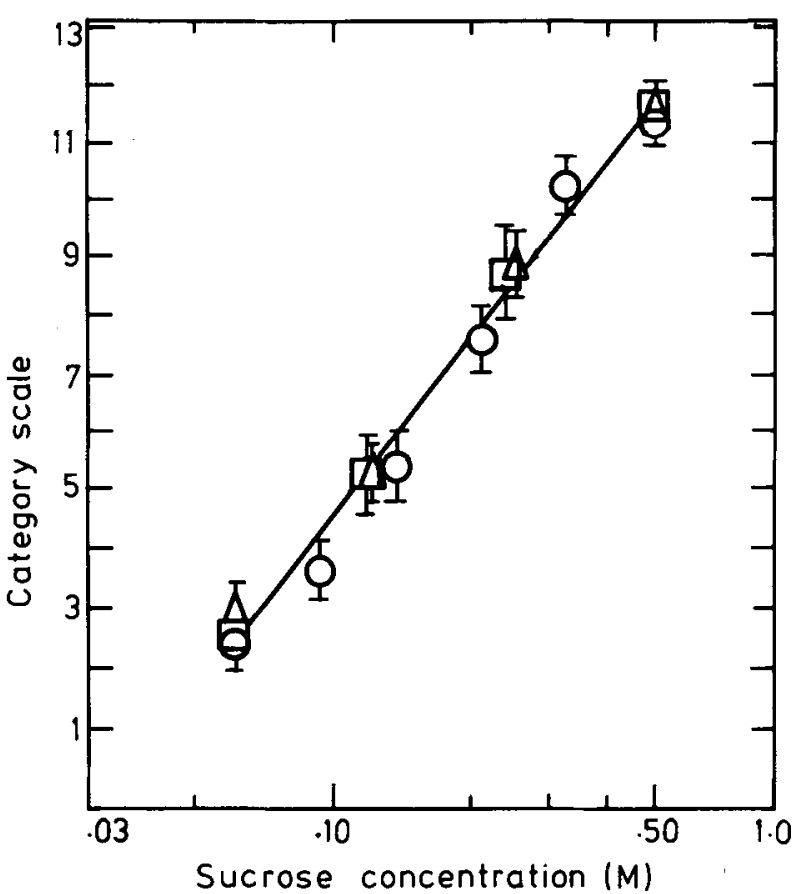

Figure 1. Mean category ratings ( $\pm 2 \mathrm{SE}$ ) of the sweetness of sucrose over the concentration range $.0625-.5000 \mathrm{M}$. The triangles, circles, and squares correspond to Parts 1 (four stimuli, geometric spacing), 2 (six stimuli, geometric spacing), and 3 (four stimuli, iterated arithmetic spacing), respectively. The straight line is the regression of ratings on log sucrose concentration.

less of sweetness magnitude. Furthermore, in each part the mean ratings are approximately equidistant on the ordinate, as required of a pure category scale.

Figure 2 gives the mean ratings for each session of the iterated Part 3. It is evident here that, although

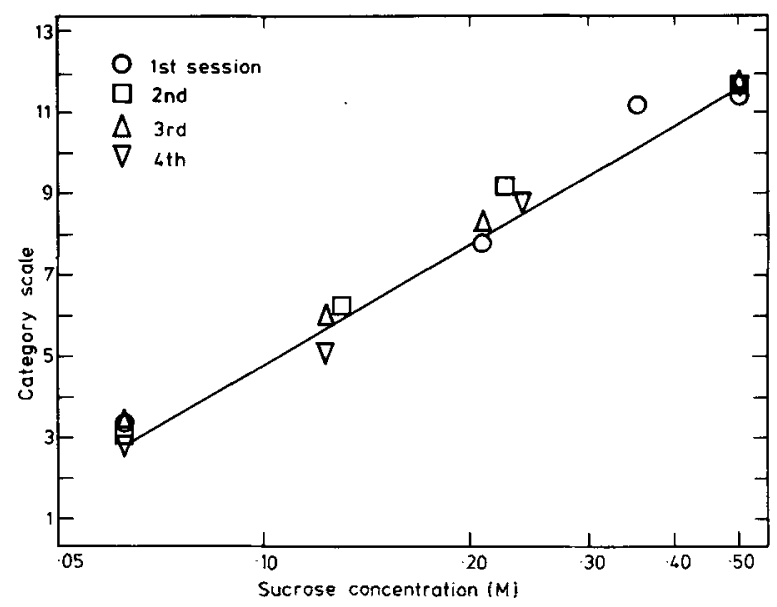

Figure 2. Mean category ratings for the sweetness of sucrose obtained using the iterative technique. The straight line is the regression curve shown in Figure 1. 
the iterative procedure was effective in producing mean ratings that are equidistant on the ordinate as required of a pure scale, the shape of the psychophysical function barely changed over the course of the four sessions. This absence of stimulus spacing bias implies that subjects made each sweetness judgment in an absolute, rather than relative, manner, unaffected by the context of the other stimuli.

For fructose, the mean ratings from the three sessions are shown in Figure 3. The variability in the ratings was similar to that in Part 3 of the sucrose experiment. As it happened, with fructose the geometric stimulus spacing produced the pure category scale at the very first session, necessitating no change in stimulus concentrations; that is, the two successive sessions were merely replications of the first. The mean ratings in Figure 3 confirm the excellent reproducibility found with sucrose and also indicate that, as for sucrose, the sweetness of fructose conforms closely to Fechner's law over the concentration range used. The ratings were fitted against log sucrose concentration by the method of least squares.

The case for glucose, however, was slightly different. Figure 4 shows that the initial geometric stimulus spacing produced mean category ratings which were not equidistant on the ordinate, thereby failing the requirement of a pure scale. Nevertheless, the next iteration had the desired effect, and it was not necessary to further adjust the concentrations for the third session. The pattern of response variability was similar to that in Part 3 of the sucrose experiment.

The deviation from linearity at low concentration suggests a systematic departure from Fechner's law; therefore the response curve was fitted to the data by eye. As for the iterated sucrose trial (Figure 2), the psychophysical function for glucose suggested at the first

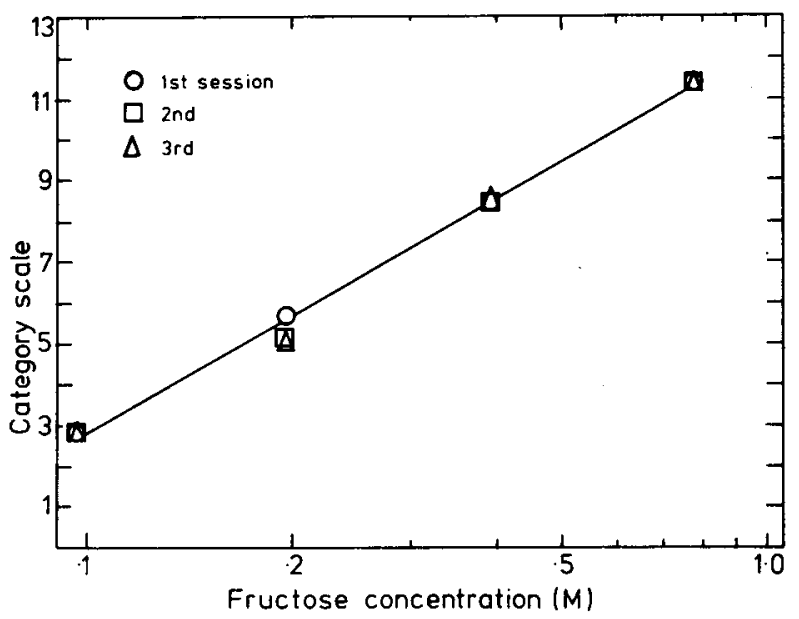

Figure 3. Mean category ratings of the sweetness of $D$ fructose over the concentration range $.0972-.7776 \mathrm{M}$, obtained by the iterative technique. The straight line is the regression of ratings on $\log$ fructose concentration.

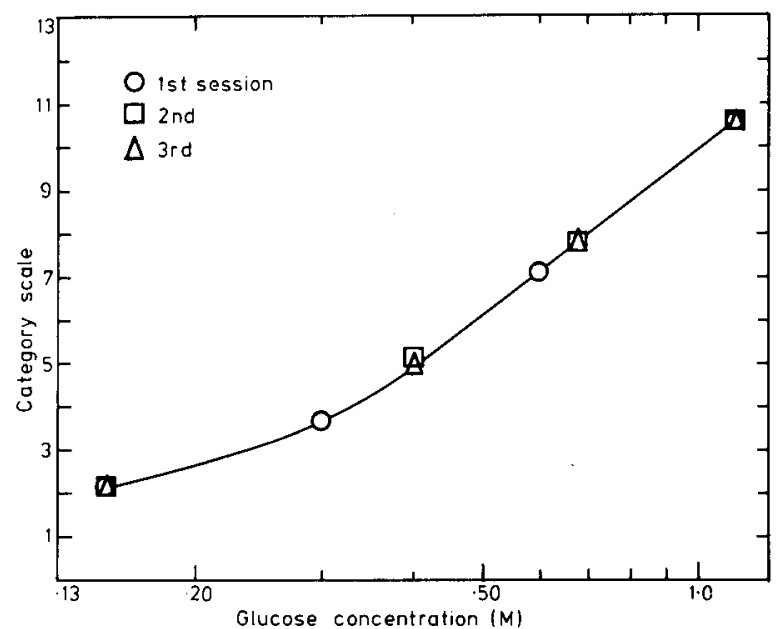

Figure 4. Mean category ratings of the sweetness of Dglucose over the concentration range $.1500-1.2000 \mathrm{M}$, obtained by the iterative technique. The curve was fitted by eye.

session is no different from that suggested after iteration: Once again, it appears that subjects made their judgments in an absolute manner, unaffected by immediate context.

Two reasons have been suggested for this absence of stimulus spacing bias (McBride, 1983). First, with only four stimuli presented at each session, there actually is less "context" than in most psychophysical experiments, and the stimulus distribution is less likely to affect judgment. Second, the verbal descriptors on the response scale may provide a constant frame of reference, thereby allowing each stimulus to be assessed independently.

To facilitate interstimulus comparison of sweetness, the psychophysical functions for the three sweeteners were redrawn in a single plot, using percent weight per volume $(\% \mathrm{w} / \mathrm{v})$ as the unit of concentration. These functions, shown in Figure 5, suggest that the sweetnesses of sucrose and fructose are almost linearly related (although there is a slight suggestion that the curves may converge at high concentration), but that, on a weight basis, fructose is sweeter than sucrose over the concentration range tested. Glucose, on the other hand, is less sweet than sucrose over the whole concentration range, and its sweetness is nonlinearly related to that of sucrose. This disparity is most pronounced at low concentration, but diminishes to some extent as concentration increases.

These findings are in good agreement with the study by Schutz and Pilgrim (1957). Using geometric stimulus spacing and a 9-point category scale, also with verbal descriptors, Schutz and Pilgrim obtained psychophysical functions for sucrose, fructose (levulose), and glucose (dextrose) that are very similar in shape to those in Figure 5 ; that is, in a semilog plot the curves for sucrose and fructose are straight lines, while the glucose function is curvilinear. In a comparison of the sweetness of sucrose 


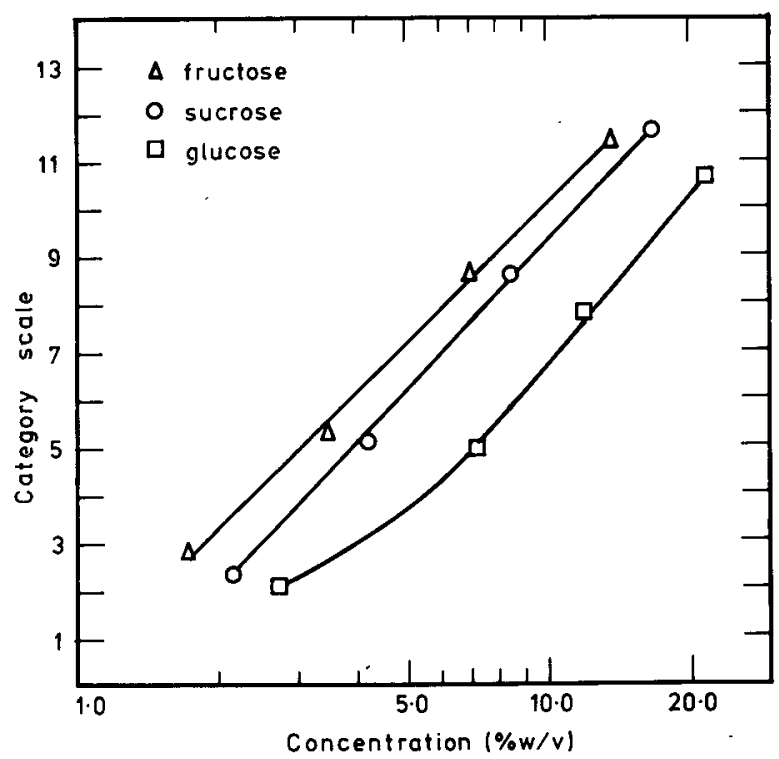

Figure 5. Comparison of the category scales for sucrose, fructose, and glucose, with concentration in percent weight per volume $(\% \mathrm{w} / \mathrm{v})$. The data points represent the stimulus spacing suggested by the iterative technique, and required of a pure scale.

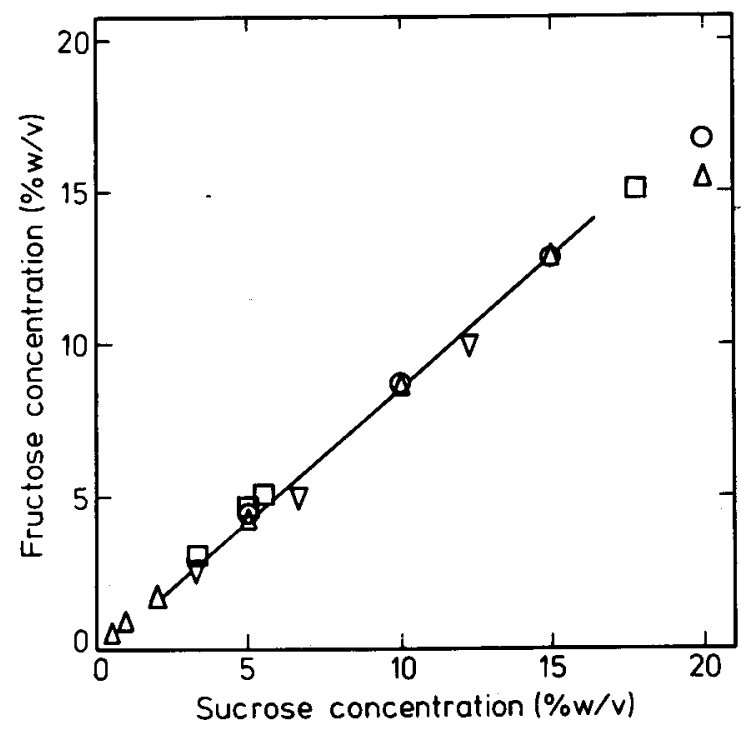

Figure 6. Comparison of the sweetness-matching function for sucrose and fructose predicted by category scaling in the present study (curve), and the results of four different studies in which sweetness matching was actually the task used-open squares (Cameron, Note 1), open circles (Dahlberg \& Penczek, Note 2), triangles (Pangborn, 1963), and inverted triangles (Yamaguchi et al., 1970).

and fructose using magnitude estimation, Cardello, Hunt, and Mann (1979) also found fructose to be sweeter than sucrose, but with the sweetness differential diminishing at high concentration. This finding con- firms the slight convergence of the sucrose and fructose curves in Figure 5.

\section{Comparison with Sweetness-Matching Studies}

The results of sweetness-matching studies are expressed in physical units (usually $\% \mathrm{w} / \mathrm{v}$ ). To facilitate direct comparison with these studies, the data curves in Figure 5 were converted accordingly. The sucrose curve was taken as the baseline, and the concentrations of fructose and of glucose which produced equivalent sweetness to a given concentration of sucrose were plotted on the ordinate. For example, Figure 5 shows that the sweetness of a $5 \%$ sucrose solution was rated as 6.2 ; the concentrations of fructose and glucose which produced the same rating are $4.1 \%$ and $8.8 \%$, respectively. In this way, predicted matching functions were obtained over the range of sucrose used, and they are shown by the curves in Figures 6 and 7.

The data points in Figures 6 and 7 are taken from the published studies available on sweetness-matching. Methodology among studies varied considerably. Yamaguchi et al. (1970) used a large taste panel (25 or 50 subjects), the method of constant stimuli as the psychophysical technique, and probit analysis for statistical interpretation; Pangborn (1963) also used the method of constant stimuli, but with 12 subjects and linear regression for statistical analysis; Cameron (Note 1) used a

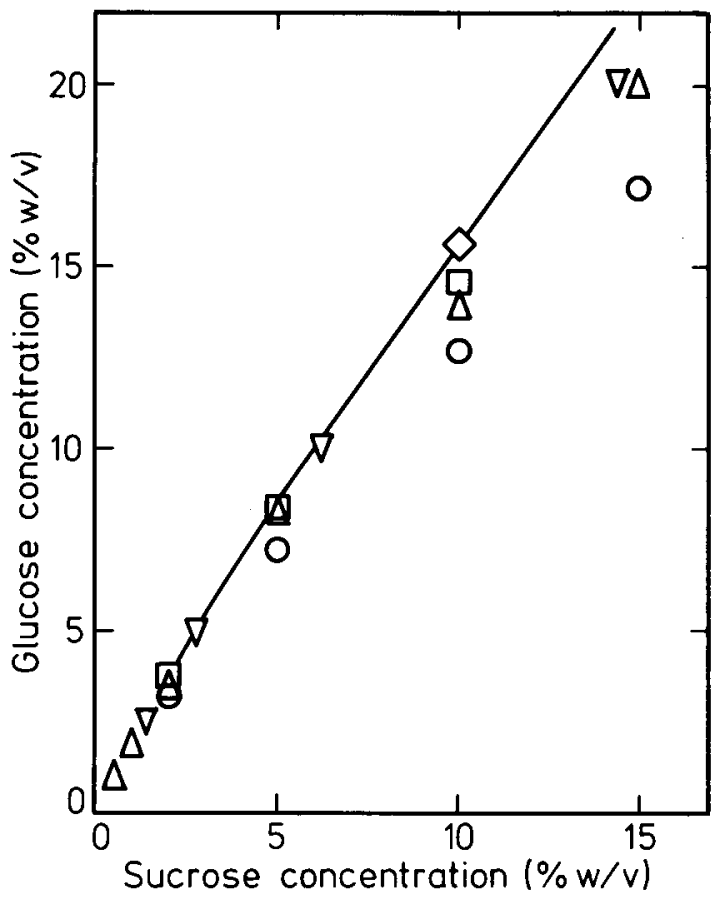

Figure 7. Comparison of the sweetness-matching function for sucrose and glucose predicted by category scaling in the present study (curve) and the results of five different studies in which sweetness matching was actually the task used. Symbols correspond to those studies cited in Figure 6, except for the diamond (from Lichtenstein, 1948). 
triad-ranking method with 15 subjects and obtained sweetness matches by linear interpolation; Dahlberg and Penczek (Note 2) used a similar technique with only three subjects; and Lichtenstein (1948) used a modified method of limits with five subjects, the "equal" response serving as the matching criterion. Notwithstanding these procedural discrepancies, there is good agreement between studies, especially for sucrose-fructose matching (Figure 6), suggesting that sweetness-matching is a robust, reliable technique. There is more variability between studies of sucrose-glucose matching (Figure 7), possibly due to the qualitatively more diffuse character of glucose sweetness.

It is clear from Figure 6 that there is excellent concordance between the predicted curve and the actual matching data. In Figure 7, the predicted function for sucrose-glucose matching is also in good agreement with the matching data; however, it does lie slightly above the data points at higher concentrations, suggesting that perhaps the sweetness of glucose was slightly underestimated in the category rating task. Assuming that sweetness matching is an unbiased procedure, the results of the present study are in accord with the contention of functional measurement analysis: Category rating can serve as a valid response measure, provided precautions are taken to preclude methodological bias.

\section{REFERENCE NOTES}

1. Cameron, A. T. The taste sense and the relative sweetness of sugars and other sweet substances (Sci. Rep. No. 9). New York: Sugar Research Foundation, 1947.

2. Dahlberg, A. C., \& Penczek, E. S. The relative sweetness of sugars as affected by concentration (Tech. Bull. No. 258). Geneva, N.Y: New York State Agricultural Experiment Station, 1941.

\section{REFERENCES}

Anderson, N. H. Functional measurement and psychophysical judgment. Psychological Review, 1970, 77, 153-170.

Anderson, N. H. Cross-task validation of functional measurement. Perception \& Psychophysics, 1972, 12, 389-395.

ANDERSON, N. H. Foundations of information integration theory. New York: Academic Press, 1981.

Anderson, N. H. Methods of information integration theory. New York: Academic Press, 1982.

Cardello, A. V., Hunt, D., \& Mann, B. Relative sweetness of fructose and sucrose in model solutions, lemon beverages and white cake. Journal of Food Science, 1979, 44, 748-751.

Lichtenstein, P. E. The relative sweetness of sugars: Sucrose and dextrose. Journal of Experimental Psychology, 1948, 38, 578-586.

MCBRIDE, R. L. Range bias in sensory evaluation. Journal of Food Technology, 1982, 17, 405-410.

McBride, R. L. A JND-scale/category-scale convergence in taste. Perception \& Psychophysics, 1983, 34, 77-83.

Pangborn, R. M. Relative taste intensities of selected sugars and organic acids. Journal of Food Science, 1963, 28, 726-733.

Pangbonn, R. M. A critical review of threshold, intensity and descriptive analysis in flavor research. In P. Schreier (Ed.), Flavour '81. Berlin: de Gruyter, 1981.

Pollack, I. Iterative techniques for unbiased rating scales. Quarterly Journal of Experimental Psychology, 1965, 17, 139-148.

Poulton, E. C. Models for biases in judging sensory magnitude. Psychological Bulletin, 1979, 86, 777-803.

Schiffman, S. S., Reilly, D. A., \& Clark, T. B., III. Qualitative differences among sweeteners. Physiology \& Behavior, $1979,23,1-9$.

Schutz, H. G., \& Pilgrim, F. J. Sweetness of various compounds and its measurement. Food Research, 1957, 22, 206-213.

Stevens, S. S., \& Galanter, E. H. Ratio scales and category scales for a dozen perceptual continua. Journal of Experimental Psychology, 1957, 54, 377-411.

WEISS, D. J. The impossible dream of Fechner and Stevens. Perception, 1981, 10, 431-434.

Yamaguchi, S., Yoshikawa, T., Ikeda, S., \& Ninomiya, T. Studies on the taste of some sweet substances. Part I. Measurement of the relative sweetness. Agricultural and Biological Chemistry, 1970, 34, 181-186. 